\title{
Effectiveness of Competitive Memory Training (COMET) for low self- esteem in youth with Autism Spectrum Disorder: A randomized controlled pilot study
}

\author{
Stella Balci', Marga Kwakman 2 , \& Bianca E. Boyer ${ }^{1,3}$
}

Published online: 22 July 2020

\begin{abstract}
Background: Youth with ASD are at risk to develop low self-esteem, which is related to both co-occurring internalizing- and externalizing problems. In this RCT $(N=24)$ we aimed to test the efficacy of Competitive Memory Training (COMET) for low self-esteem in youth with ASD (8-16y). Method: We compared the combination of COMET and Care As Usual (COMET+CAU) with CAU-only, to explore whether COMET had additional effects on low self-esteem and co-occurring symptoms of youth with ASD. Stability of effects was measured seven weeks later. Results: Participants receiving COMET $+\mathrm{CAU}$ showed greater improvement on parent-reported self-esteem and externalizing symptoms than participants receiving CAUonly. Similar improvements between groups were found on self-reported self-esteem and depressive symptoms. Improvements remained stable until seven weeks after having received COMET $+\mathrm{CAU}$, with depressive symptomatology improving even further. Conclusions: Given the small sample size and mixed results, this pilot study does not allow us to declare COMET as being necessary in enhancing low selfesteem in ASD. However, this study indicates that when given parallel to CAU, COMET can help to improve self-esteem and co-occurring externalizing problems in youth with ASD in only a short period of time.
\end{abstract}

Keywords Autism Spectrum Disorder • Self-esteem • Youth • Cognitive Behavioral Therapy (CBT) • Pilot Study

Autism spectrum disorders (ASD) are pervasive neurodevelopmental disorders, affecting 1 in 160 children worldwide (World Health Organization, 2017). The core features that characterize individuals within this spectrum are deficits in social communication and interaction as well as the presence of repetitive patterns of behavior (American Psychiatric Association [APA], 2013). Several underlying mechanisms have been proposed that can cause individuals with ASD to process information differently from others, such as a weaker central coherence, delayed development of a Theory of Mind and difficulties in executive functioning, such as planning, inhibition and flexibility (Frith, 2003, MacMullen-Freeman, Locke, Rotheram-Fuller \& Mandell, 2017; Ozonoff \& Jensen, 1999; Ozonoff, Strayer, McMahon \&
Filloux, 1994; Schuh \& Eigsit, 2012). In addition, some individuals with ASD show difficulties in the development of active or passive language, show discrepancies between different intellectual abilities or show co-occurring motor disabilities (Kwok, Brown, Smyth, \& Cardy, 2015; Joseph, TagerFlusberg, \& Lord, 2002; Ankenman, Elgin, Sullivan, Vincent, \& Bernier, 2014; Ming, Brimacombe, \& Wagner, 2007; Fournier, Hass, Naik, Lodha, \& Cauraugh, 2010).

\footnotetext{
${ }^{1}$ Department of Developmental Psychology, University of Amsterdam, The Netherlands

${ }^{2}$ Youz Parnassia Groep, Purmerend, The Netherlands

${ }^{3}$ Psychologenpraktijk Kuin, Haarlem, The Netherlands
}

Corresponding author Dr. B. E. Boyer, Department of Developmental Psychology, University of Amsterdam, Nieuwe Achtergracht 129B, 1001 NK Amsterdam, The Netherlands. Email: b.e.boyer@uva.nl 
As society greatly appeals to these functions and skills, some children and adolescents with ASD encounter challenges in their academic careers, in developing friendships or in sports (APA, 2013; Estes, Rivera, Bryan, Cali, \& Dawson, 2011). As youth with ASD get older, they become more aware about these differences between them and their typically developing peers (Portway \& Johnson, 2003). Being confronted with these differences might interfere with the development of a positive self-esteem (Mann, Hosman, Schaalma, \& de Vries, 2004).

Self-esteem can be defined as a global evaluation of one's own competencies. It is about recognizing one's own strengths and weaknesses in different areas, resulting in an overall self-worth; which reflects self-esteem (Harter, Waters, \& Whitesell, 1998). Children evaluate themselves based on comparison to others and on feedback from others (Mann et al., 2004). In early childhood, feedback is mainly given by parents, but as children grow older, teachers and peers get a more prominent role in children's lives and their feedback becomes more important for self-evaluation (Harter, 2012). These comparisons to and feedback from others can impact their self-esteem (Harter, 2012; Mann et al., 2004; Westenberg, Drewes, Goedhart, Siebelink, \& Treffers, 2004). The vulnerability hypothesis of self-esteem states that low self-esteem might be predictive of developing psychopathology such as depression and anxiety (Van Tuijl et al. 2014). This is supported by research in normative populations, showing associations between low self-esteem and development of depression, anxiety and overall lower well being (Hawker \& Boulton, 2000; Sowislo \& Orth, 2013).

Only a scarce number of studies have focused on self-esteem evaluations of youth with ASD. One could imagine that facing challenges on a broad scale of domains might make youth with ASD become more attentive to their shortcomings as compared to their positive characteristics and competencies, which in turn could hinder the development of positive self-esteem. Indeed, the only two studies on self-esteem in youth with ASD showed that they maintain a low self-esteem compared to typically developing peers on parentreports and self-reports (McCauley et al., 2017; Van der Cruijsen \& Boyer, under revision). Moreover, in these studies low self-esteem in youth with ASD appeared to be related to both co-occurring internalizing- and externalizing problems. Accordingly, existing research confirms that cooccurrence of psychopathology exists among youth with ASD, with depression and anxiety being among the most common co-occurring disorders (Matson \& Nebel-Schwalm, 2007; Simonoff et al., 2008; White, Oswald, Ollendick, \& Scahill, 2009). These co-occurring problems add to the consequences of having ASD for the child itself, their parents and their family. For example, they report lower quality of life (Broquere et al., 2016), more depressive symptoms and parental stress in parents (Lee, Harrington, Louie, \& Newschaffer, 2008; Van Steijn, Oerlemans, Van Aken, Buitelaar, \& Rommelse, 2014). Since ASD is a chronic disorder and symptoms can be improved but not cured, treatment of this low self-esteem seems promising to make these children and adolescents feel better about themselves and prevent the development of co-occurring disorders.

As a low self-esteem is a common factor in disorders affecting social and emotional functioning, Competitive Memory Training (COMET) has been developed as a transdiagnostic treatment to focus solely on this concept (Korrelboom, Van der Weele, Gjaltema, \& Hoogstraten, 2009). COMET is a cognitive behavioral therapy (CBT) that uses contraconditioning to challenge negative thoughts and feelings about the self and formulate new, more nuanced, positive ones. COMET attempts to make these alterations by reaching out to different cognitive- and affective domains within the brain, to include the whole network involved: thoughts, feelings, bodily posture, facial expressions and auditory elements (Lang, 1985). So far, COMET has been found successful in enhancing self-esteem in adults within several clinical populations, among which depressive disorders (Ekkers et al., 2011; Korrelboom, Maarsingh, \& Huijbrechts, 2012), anxiety disorders (Staring et al., 2016), obsessivecompulsive disorders (Schneider, Wittekind, Talhof, Korrelboom, \& Moritz, 2015), eating disorders (Korrelboom, De Jong, Huijbrechts, \& Daansen, 2009) and schizophrenia (Van der Gaag, 
Van Oosterhout, Daalman, Sommer, \& Korrelboom, 2012). As treatments aiming on selfesteem were lacking for youth, in 2014 COMET has been adapted for treatment of children and adolescents (Kuin \& Peters, 2014).

Several changes have been made to the COMET protocol for youth, in order to make the intervention more age appropriate (Kuin \& Peters, 2014). The main differences are concerned with the formulation of exercises and homework: tasks and assignments are formulated more explicit, which makes it easier for children and adolescents to understand how to carry them out. For example, whereas adults are asked to activate positive emotions, children/adolescents are asked to recall a positive memory in order to activate positive emotions. The treatment protocol is only 7 individual sessions and is therefore easy to implement in mental health care for youth. A first pilot study showed promising results in the effectiveness of COMET for improving self-esteem in youth with low self-esteem $(N=32$, Kuin, Van Steensel, \& Bögels, in preparation). In a nonrandomized trial, the authors found pre- to posttreatment improvements in self-reported selfesteem $(d=0.35-0.69)$, depressive symptomatology $(d=0.53)$ and internalizing behavior $(d=0.37)$.

So far, no study has yet been conducted to assess whether COMET is also effective for enhancing self-esteem in youth with ASD. Since the vulnerability hypothesis states that low self-esteem is predictive of the development of psychopathology, treating their self-esteem could therefore simultaneously decrease co-occurring psychopathology. The current pilot study explored the efficacy of COMET in youth with ASD aged 8 to 16 years. We do not seek to prove COMET superior to any other intervention: as ASD is a chronic disorder with a heterogeneous range of cooccurring problems, we do not expect COMET to be sufficient as stand-alone intervention for families with a child with ASD. Rather, we want to investigate whether COMET has additive effects to Care As Usual (CAU) on primary (self-esteem and self-worth) and secondary (co-occurring internalizing and externalizing symptoms) outcome measures when given in combination with $\mathrm{CAU}$ (COMET+CAU). Our two hypotheses were: 1)
When given COMET + CAU, self-esteem and selfworth (primary outcome measures) of youth with ASD will improve and co-occurring internalizing and externalizing symptomatology will decrease from pretest to posttest, more than when given CAU alone; 2) Effects of COMET $+\mathrm{CAU}$ will remain stable or improve further to seven weeks after treatment.

\section{Methods}

\section{Trial Design}

This was a multicenter ( 3 sites), two-arm parallel pilot study, with equal randomization [1:1]. Participants were recruited between February 2017 and February 2019; follow-up assessments were between June 2017 and June 2019. The study was approved by the Ethics Committee of the University of Amsterdam (2016-DP-7315) and the study was preregistered in the Clinical Trials Registration (\#25615) in December 2016. No major changes were made to the trial design afterwards.

\section{Participants and Procedure}

This pilot study was advertised at participating mental health institutions. When interested, youth and their parents could apply to participate. To be included in this study, children and adolescents had to meet the following criteria: (1) a diagnosis of ASD, by an independent certified psychologist or child psychiatrist based on the DSM-IV-TR or DSM-5 (APA, 2000, 2013), (2) a confirmation of ASD based on the Dutch Social Responsiveness Scale (SRS-2). The SRS-2 is a 65-item parentreported questionnaire aimed at detecting interpersonal, communicative and stereotype behaviors that are typical for ASD (Roeyers, Druart, De Schryver, \& Schittekatte, 2011). Every item has a four-point Likert Scale (1= 'strongly agree' to $4=$ 'strongly disagree'), and a higher score indicates the presence of more ASD characteristics. Children were included if they reached a T-score of 61 or above, which indicates the presence of at least mild to moderate autism symptoms, (3) aged between 8 and 16 years, (4) a stated low self-esteem 
by the child/adolescent and/or parent, (5) a full scale IQ (FSIQ) $>80$ measured by the short version of the Dutch Wechsler Intelligence Scale for Children (WISC-III, Kort et al., 2005). The short version consists of two subtests of the WISC-III: Block design and Vocabulary. The norm scores of these two subtests correlate highly with FSIQ (Sattler, 2001), (6) sufficient understanding of the Dutch language by both the child and parent,
(7) sufficient motivation for treatment, (8) Completion of all three assessments of this study. Participants were excluded if: (1) severe depressive disorder with suicidal thoughts/ideations was diagnosed, and (2) low self-esteem was due to traumatization of recent events or ongoing traumatization of past events. Based on ethical considerations, when any of these exclusion criteria was present an alternative intervention was more appropriate.

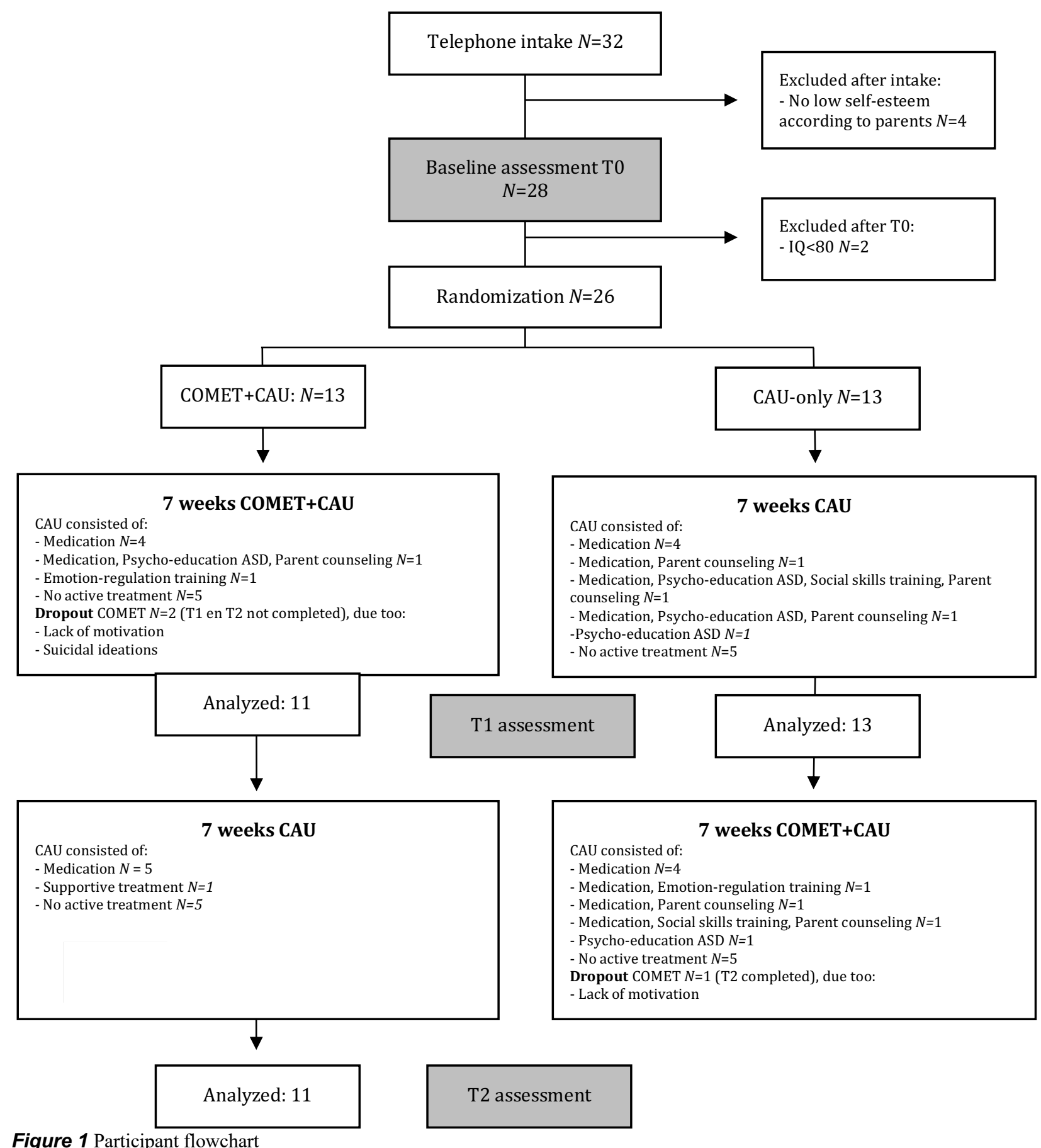

Figure 1 Participant flowchart 
Before participation, written informed consents were acquired from all participants and their parents. The first assessment took place at baseline (T0). Due to ethical considerations all participants received COMET $+\mathrm{CAU}$. To be able to compare the COMET $+\mathrm{CAU}$ to a CAU only condition, we divided our total sample into two conditions and counter-balanced the order of received treatments: one group first received $\mathrm{COMET}+\mathrm{CAU}$ for 7 weeks (from now on called COMET $+\mathrm{CAU}$ condition), while the second group first received CAU-only for 7 weeks (from now on called CAU-only condition). After these 7 weeks T1 assessment took place and the COMET $+\mathrm{CAU}$ condition received CAU only for 7 weeks, whereas the CAU-only condition received COMET $+\mathrm{CAU}$ for 7 weeks (see Figure 1 for trial design and participant flow) after which T2 assessment took place. Parent-reported questionnaires were at all time assessments completed by the primary caregiver. Allocation of the participants took place after baseline assessment, using a computer-based algorithm for equal randomization [1:1]. Interventions and assessments were all administered at the participants own outpatient mental health institute.

Thirty-two participants applied for the study, of which 28 were included based on the in- and exclusion criteria. All 28 completed baseline assessment; two participants were excluded from the study because they had FSIQ's below 80 . Therefore 26 participants were randomly assigned to the two conditions.

\section{Treatments}

\section{Competitive Memory Training (COMET)}

COMET is a cognitive behavioral therapy based on Brewin's contra-conditioning principle (2006). The purpose of the intervention is to establish and strengthen new positive networks concerning thoughts and feelings about the self, in opposition to existing dysfunctional ones. Not only thoughts and feelings are involved in doing so; facial expressions and bodily postures (Holmes, Mathews, Dalgleish, \& Mackintosh, 2006), self-verbalization (Posthuma \& Lange, 1999) and music (Van der Does, 2002) are also used. By involving all these different elements, successful alteration of the whole emotional network can be maintained (Lang, 1985). In addition to the original COMET protocol, positive-attention training elements are implemented into the COMET youth protocol. Focusing attention on positive experiences facilitates the construction of positive networks in youth with low self-esteem (Reijntjes, Thomaes, Kamphuis, Orobio de Castro, \& Telch, 2010). Therefore, positive attention training was administered during sessions and homework, consisting of exercises meant to focus attention on positive experiences and characteristics. Homework was given after every session to generalize learned skills to daily life. At the start of each new session homework was discussed. Each individual session (approximately $45 \mathrm{~min}$.) is focused on a main topic, summarized in Table 1. In one parental session, parents are taught to focus on positive elements and behaviors of the child, to compliment the child at moments of accomplishment and to ignore negative behavior. More information on the treatment protocol can be found at the publisher (Kuin \& Peters, 2014).

Table 1 COMET session description

\begin{tabular}{ll}
\hline Session & Treatment goal \\
\hline 1 & $\begin{array}{l}\text { Identification of automatic negative thoughts } \\
\text { about the self }\end{array}$ \\
2 & $\begin{array}{l}\text { Finding incompatible positive thoughts and } \\
\text { characteristics }\end{array}$ \\
3 & $\begin{array}{l}\text { Strengthening positive self-esteem by positive } \\
\text { memories and self-verbalization }\end{array}$ \\
Parent & $\begin{array}{l}\text { Establishing a positive environment for the child } \\
4\end{array}$ \\
5 & $\begin{array}{l}\text { Strengthening positive self-esteem by facial } \\
\text { expression, bodily posture and music }\end{array}$ \\
& $\begin{array}{l}\text { Strengthening positive self-esteem by positive } \\
\text { memories and comparison to others }\end{array}$ \\
6 & $\begin{array}{l}\text { Overcoming triggers of negative self-thought } \\
\text { Acquisition of a functional coping strategy for }\end{array}$ \\
\hline
\end{tabular}

From: Kuin \& Peters, 2014

\section{Care As Usual}

For each participant Care As Usual (CAU) was chosen and administered by their own therapist. The 
reason we did not decide to implement only one and the same form of CAU for all participants was primarily an ethical one: it would not be ethical to restrain participants from an intervention that would be more useful for them than the one we would have chosen. Since we are not trying to compare COMET with a specific form of CAU, neither trying to reveal COMET as stand-alone intervention as being superior to a certain $\mathrm{CAU}$, there was no need to have a fixed CAU. Moreover, our goal is to see whether COMET has beneficiary effects when given parallel to whatever kind of CAU. In addition, including different CAU's in our sample allows us to be able to generalize findings to the CAU as given outside the lab setting. Interventions that were used as CAU are summarized per group in Figure 1 and contained social skills training, emotion regulation training, psycho-education on ASD, family counseling, parent counseling and medication use. Of the 12 analyzed participants $(50 \%)$ that used medication, 6 used Methylphenidate alone, 3 used Aripiprazole and Methylphenidate, 1 used Aripiprazole and Levothyroxine, 1 used Fluoxetine and Risperidone and 1 used Dexamphetamine and Risperidone.

\section{Outcome Measures}

\section{Primary outcome measures}

Self-esteem was measured using the Rosenberg Self-Esteem Scale (RSES; Rosenberg, 1979; Everaert et al., 2010). In this study three versions were used; for children between 8 and 12 the RSES-child version was administered, for adolescents between 12 and 18 the RSESadolescent version and for parent-report on the child's self-esteem the RSES-parent version. With each questionnaire, the original questions were administered, only wording changed with informant perspective. For instance, for children items were formulated as following "I am able to do things as well as most other children", for adolescents "I am able to do things as well as other adolescents" and for parents "My child is able to do things as well as other children". The RSES consists of 10 items, each having a four-point Likert scale. Scores range between 10 and 40, with scores under 26 indicating low self-esteem (Isomaa, Väänänen, Fjörd, Kaltiala-
Heino, \& Marttunen, 2013). The RSES has previously been proven sufficiently reliable and valid (Blascovich \& Tomaka, 1991). It has been used in a wide range of studies concerning selfesteem (Schmitt \& Allik, 2005). In our sample, RSES reports showed a high internal consistency at all three assessments, ranging from Cronbach's $\alpha=$ $0.90-0.95$ for children, $\alpha=0.84-0.93$ for adolescents and $\alpha=0.73-0.93$ for parents.

Self-worth was measured using the selfworth scale of the Self Perception Profile for Children (SPPC; Harter, 1985; Veerman, Straathof, Treffers, Van den Bergh, \& Ten Brink, 1997): a self-reported questionnaire for children aged between 8 and 12 years. For adolescents of 12 years and older, the self-worth scale of the Self Perception Profile for Adolescents (SPPA; Harter, 1988; Treffers, Goedhart, Veerman, Van den Bergh, Ackaert, \& De Ryck, 2002) was used. The selfworth scale of the SPPC and SPPA contain respectively 6 and 5 items. Each item consists of two statements regarding self-esteem states (e.g. "Some children are often disappointed in themselves, but others are almost never disappointed in themselves"). The participant has to choose what statement is most true for him/her and to what extent. Percentile scores on the subscales were calculated and computed into one new variable as to treat children and adolescent as one group in order to use for main analysis. Higher scores indicate higher perceived self-worth. In our sample, internal consistency was high for both the SPPC and the SPPA, respectively ranging from Cronbach's $\alpha=0.83-0.94$ and Cronbach's $\alpha=0.74$ 0.90 over all 3 assessments.

\section{Secondary outcome measures}

Depressive symptoms were measured using the Child Depression Inventory (CDI): a 27-item selfreported questionnaire (Sitarenios \& Kovacs, 1999; Timbremont \& Braet, 2002). Each item consists of three statements regarding the state of mood, with each statement reflecting another score. Participants have to report what statement suits them best. For example, "1) Sometimes I am sad, 2) Often I am sad or 3) I am always sad." Scores range from 0 to 54, with higher scores indicating more depressive symptoms. The CDI has previously been proven 
sufficiently reliable, with Cronbach's $\alpha$ ranging from 0.80 to 0.94 (Saylor, Finch, Spirito, \&Bennett, 1984). In our sample, CDI reports showed high internal consistency, ranging from Cronbach's $\alpha=0.79-0.86$ over all 3 assessments .

Internalizing and externalizing problems are measured using the Child Behavior Checklist (CBCL): a 112-item parent-report questionnaire on behavioral and emotional functioning of their child (Achenbach, 1994; Verhulst, Van der Ende, \& Koot, 1996). The CBCL can be divided into two broadband scales, reflecting internalizing- and externalizing behaviors. Items contain a statement about which parents have to indicate to what extent it applies to their child, using a 3-point Likert Scale $(0=$ not true all, $1=$ somewhat $/$ sometimes true, $2=$ often true). For example: "Behaves too young for his/her age". Higher scores indicate more internalizing and externalizing problems. In our sample, CBCL reports showed high internal consistency for both the internalizing and externalizing broadband scales, respectively ranging from Cronbach's $\alpha=0.88-0.90$ and $\alpha=0.81-$ 0.86 over all 3 assessments.

\section{Therapists and Treatment Fidelity}

In total, 20 therapists participated in the study, in 3 different outpatient locations. All therapists had at least a master's degree in psychology. The number of children that was treated by each therapist varied from 1 to 4 . To elevate treatment fidelity of COMET, several actions were taken: two authors (BB and $\mathrm{MK}$ ) trained all therapist in the treatment protocol in a 3-hour training, in which they also emphasized the need for treatment fidelity. In addition, COMET is described in a treatment manual that is distributed over all treatment locations (Kuin \& Peters, 2014). Finally, all treatments are executed using the COMET workbook to follow the same structure and order of assignments. To rate their treatment adherence, therapists were asked to fill in a registration book that asked them to rate whether they executed all assignments and whether homework was completed by the participant in the workbook as it is described in the treatment manual (yes/no). Based on these registration books, percentages of addressed elements of COMET and completion of homework assignments were calculated.

\section{Statistical Analysis}

Attrition took place in both conditions $($ COMET + CAU $N=2$, CAU-only $N=1)$, due to lack of motivation $(N=2)$ and suicidal ideation $(N$ $=1)$. Of these 3 dropouts $(11.54 \%)$, one completed all assessments, leaving 24 participants for main analyses. A-priori power analysis revealed that to reach a power of 0.80 , a sample size of $N=68$ was required. However, due to low participant recruitment we unfortunately were not able to do so. The results derived from this study should therefore be interpreted with caution. All analyses in this study have been conducted with SPSS version 24.0.

First, we compared the groups on demographic characteristics at baseline using independent samples t-tests and chi-square tests, to assure successful randomization. Then we compared the groups on all outcome variables at baseline using a MANOVA, followed up by univariate tests. To test our main hypothesis, whether participants in the COMET $+\mathrm{CAU}$ condition improved more on primary and secondary outcome variables than participants in the CAUonly condition, we compared the first two measurements ( $\mathrm{T} 0$ and $\mathrm{T} 1$ ) of both conditions on all outcome variables using a repeated measures MANOVA. To analyze our second hypothesis, whether the treatment effects for participants in the COMET $+\mathrm{CAU}$ condition found between $\mathrm{T} 0$ and $\mathrm{T} 1$ remain after seven weeks at $\mathrm{T} 2$, a paired sample $\mathrm{t}$ test has been used to compare posttest to follow-up assessment. Assumptions for parametric tests were again checked for all outcome variables, and when violated, the non-parametric alternative, Wilcoxon signed-rank test, was used.

\section{Results}

Sample description and baseline comparison

Participants had a mean age of $12.00(S D=2.40)$ years, of which $79.17 \%$ was male, with an average FSIQ of $97.92(S D=12.62)$. Before running the main analyses, we checked whether participants differed on any demographic as well as outcome 
Table 2 Sample description and group comparison at baseline

\begin{tabular}{|c|c|c|c|c|}
\hline & $\begin{array}{l}\text { Total Sample } \\
(N=24) \\
M / N(S D / \%)\end{array}$ & $\begin{array}{l}\text { COMET+CAU } \\
\text { condition } \\
(N=11) \\
M / N(S D / \%)\end{array}$ & $\begin{array}{l}\text { CAU-only } \\
\text { condition } \\
(N=13) \\
M / N(S D / \%)\end{array}$ & $\begin{array}{l}\text { Group } \\
\text { comparison }\end{array}$ \\
\hline Age in years & $12.00(2.40)$ & $12.18(2.39)$ & $11.84(2.49)$ & NS \\
\hline$N$ boys $(\%)$ & $19(79.17)$ & $8(72.72)$ & $11(84.62)$ & NS \\
\hline FSIQ & $97.92(12.62)$ & $102.82(14.01)$ & $93.77(10.06)$ & NS \\
\hline SRS T-score & $75.29(9.08)$ & $74.64(10.60)$ & $75.85(7.98)$ & NS \\
\hline Medication (use) & $12(50.00)$ & $5(45.45)$ & $7(53.84)$ & NS \\
\hline Childs educational level & & & & NS \\
\hline$N$ Primary education (\%) & $13(54.17)$ & $5(45.45)$ & $8(61.54)$ & \\
\hline \multicolumn{5}{|l|}{ Secondary education } \\
\hline$N$ Low $(\%)$ & $0(0,00)$ & $0(0,00)$ & $0(0,00)$ & \\
\hline$N$ Average (\%) & $10(41.67)$ & $5(45.45)$ & $5(38.46)$ & \\
\hline$N$ High (\%) & $1(4.17)$ & $1(9.09)$ & & \\
\hline$N$ Highest (\%) & $0(0,00)$ & $0(0,00)$ & $0(0,00)$ & \\
\hline Mother's educational level & & & & NS \\
\hline$N$ Primary education (\%) & $3(12.50)$ & $1(9.09)$ & $2(15.38)$ & \\
\hline \multicolumn{5}{|l|}{ Secondary education } \\
\hline$N$ Low $(\%)$ & $3(12.50)$ & $2(18.18)$ & $1(7.69)$ & \\
\hline$N$ Average(\%) & $8(33.33)$ & $5(45.45)$ & $3(23.08)$ & \\
\hline$N \operatorname{High}(\%)$ & $9(37.50)$ & $3(27.27)$ & $6(46.15)$ & \\
\hline$N$ Highest $(\%)$ & $1(4.17)$ & & $1(7.69)$ & \\
\hline Father's educational level & & & & NS \\
\hline$N$ Primary education (\%) & $5(20.83)$ & $3(27.27)$ & $2(15.38)$ & \\
\hline \multicolumn{5}{|l|}{ Secondary education } \\
\hline$N$ Low $(\%)$ & $8(33.33)$ & $3(27.27)$ & $5(38.46)$ & \\
\hline$N$ Average (\%) & $8(33.33)$ & $3(27.27)$ & $5(38.46)$ & \\
\hline$N$ High (\%) & $3(12.50)$ & $2(18.18)$ & $1(7.69)$ & \\
\hline$N$ Highest (\%) & $0(0,00)$ & $0(0,00)$ & $0(0,00)$ & \\
\hline Omnibus test & & & & NS \\
\hline Self-reported self-esteem & $28.75(6.73)$ & $30.09(6.58)$ & $27.62(6.90)$ & NS \\
\hline Parent-reported self-esteem & $20.63(4.27)$ & $21.36(4.99)$ & $20.00(3.65)$ & NS \\
\hline Self-worth & $36.33(32.19)$ & $43.55(33.46)$ & $30.23(31.07)$ & NS \\
\hline Depressive symptoms & $14.46(6.56)$ & $12.82(3.25)$ & $15.85(8.32)$ & NS \\
\hline Internalizing symptoms & $24.63(10.53)$ & $23.73(11.83)$ & $25.38(9.72)$ & NS \\
\hline Externalizing symptoms & $14.63(7.19)$ & $12.64(7.23)$ & $16.31(9.98)$ & NS \\
\hline
\end{tabular}


variables at baseline. Group comparisons between conditions showed no differences, indicating that any differences found hereafter cannot be attributed to these baseline characteristics. Based on the cutoff scores suggested by Isomaa et al. (2013), observed parent-reported self-esteem and selfreported self-esteem at baseline indicate that on average, parents report a clinically low self-esteem $(M=20.63, S D=4.27)$, whereas children indicate normative self-esteem $\quad(M=28.75, \quad S D=6.73)$. Demographic and baseline descriptions are summarized in Table 2.

\section{Treatment Fidelity for COMET}

In the total group $88.97 \%$ completed all sessions of COMET. There were no differences $(p=0.78)$ in the average percentage of treatment fidelity of the COMET protocol, between the COMET $+\mathrm{CAU}$ condition $(M=88.24 \%, \quad S D=7.78) \quad$ and the participants in the CAU-only condition whom later received COMET $(M=89.59 \%, \quad S D=13.93)$. Likewise, $84.85 \%$ of the total group completed all homework assignments. Again, no differences $(p=0.57)$ were found in the average percentage of homework fidelity between the COMET $+\mathrm{CAU}$ condition $(M=88.43 \%, \quad S D=27.00)$ and the participants in the CAU-only condition whom later received COMET $(M=81.82 \%, S D=28.75)$.

\section{Treatment Effects: Time effect}

First, the omnibus test revealed that participants in both conditions showed improvements between $\mathrm{T} 0$ and T1 assessments, $\quad F(6,17)=3.05, \quad p=0.03$, $\eta_{p}{ }^{2}=0.52$. More specifically, self-reported selfesteem, parent-reported self-esteem, depressive symptomatology and externalizing symptoms improved over time regardless of condition, respectively $F(1,22)=7.96, \quad p=0.01, \quad \eta_{p}{ }^{2}=0.27$, $F(1,22)=8.78, \quad p<0.01, \quad \eta_{p}{ }^{2}=0.29, \quad F(1,22)=10.08$, $p<0.01, \quad \eta_{p}{ }^{2}=0.31 \quad$ and $F(1,22)=4.84, \quad p=0.04$, $\eta_{p}{ }^{2}=0.18$. No significant improvements were found for feelings of self-worth $\left(p=0.14, \eta_{p}{ }^{2}=0.10\right)$ and internalizing symptoms $\left(p=0.48, \eta_{p}{ }^{2}=0.02\right)$ over time (see Table 3 for descriptives of treatment effects).

\section{Treatment Effects: Time* Treatment interaction}

When comparing the two treatments on improvements between pre- and posttest, results were mixed. Parent-reported self-esteem, $F(1,22)=6.95, \quad p=0.02, \quad \eta_{p}{ }^{2}=0.24$, and parentreported externalizing symptoms, $F(1,22)=4.84$, $p=0.04, \eta_{p}{ }^{2}=0.18$, showed greater improvement in the COMET+CAU group as compared to the CAUonly group (see Figure 2). However, improvements were similar in both groups on self-reported selfesteem $\quad\left(p=0.18, \quad \eta_{p}{ }^{2}=0.08\right)$ and self-reported depressive symptoms $\left(p=0.77, \eta_{\mathrm{p}}{ }^{2}<0.01\right)$. And both groups showed similar lack of improvement on feelings of self-worth $\left(p=0.19, \eta_{p}{ }^{2}=0.08\right)$ and parent-reported internalizing symptoms $(p=0.27$, $\left.\eta_{\mathrm{p}}{ }^{2}=0.06\right)$.

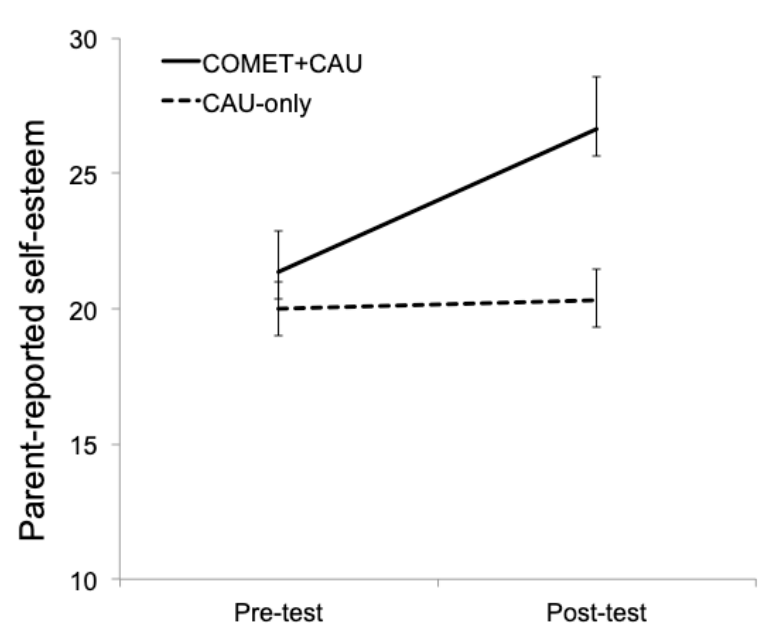

Note: CAU, Care As Usual; COMET, Competitive Memory Training

Figure 2. Parent reported self-esteem

\section{7-week follow-up}

Analyses of our second hypothesis, whether the effects for participants in the COMET+CAU condition remain after seven weeks, showed maintenance of effects on primary and secondary outcome measures. Signifying that improvements of parent-reported self-esteem $(p=0.43, d=0.20)$ and externalizing symptoms ( $p=0.93, r=0.02$ ) remained stable seven weeks after having received COMET + CAU. Self-reported depressive symptoms even decreased further after seven weeks of having received COMET $+\mathrm{CAU}, \quad t(1,10)=3.30, \quad p<0.01$, $d=0.44$, but as we have no comparison to a CAUonly group at follow-up we cannot contribute this to COMET. The improvements over time, 
independent of treatment allocation, found for selfworth ( $p=0.07, r=0.55)$ and internalizing symptoms ( $p=0.24, d=0.37$ ) were also maintained to $\mathrm{T} 2$.

Table 3 Group comparison of pre- to posttreatment improvements on primary and secondary outcome measures between COMET+CAU and CAU-only.

\begin{tabular}{|c|c|c|c|c|c|c|c|c|}
\hline & \multicolumn{2}{|c|}{$\begin{array}{l}\text { COMET+CAU } \\
N=11\end{array}$} & \multicolumn{2}{|l|}{$\begin{array}{l}\text { CAU-only } \\
N=13\end{array}$} & \multicolumn{2}{|c|}{ Time effects } & \multicolumn{2}{|c|}{$\begin{array}{l}\text { Time* Treatment } \\
\text { effects }\end{array}$} \\
\hline & $\mathrm{T} 0$ & $\mathrm{~T} 1$ & T0 & $\mathrm{T} 1$ & & & & \\
\hline & $M(S D)$ & $M(S D)$ & $M(S D)$ & $M(S D)$ & $p$ & $\eta_{p}{ }^{2}$ & $p$ & $\eta_{p}^{2}$ \\
\hline Omnibus test & & & & & 0.03 & 0.52 & 0.14 & 0.40 \\
\hline $\begin{array}{l}\text { Self-reported } \\
\text { self-esteem }\end{array}$ & $30.09(6.58)$ & $34.18(6.79)$ & $27.62(6.90)$ & $29.00(7.52)$ & 0.01 & 0.27 & 0.18 & 0.08 \\
\hline $\begin{array}{l}\text { Parent-reported self- } \\
\text { esteem }\end{array}$ & $21.36(4.99)$ & $26.64(6.41)$ & $20.00(3.65)$ & $20.31(4.23)$ & $<0.01$ & 0.29 & 0.02 & 0.24 \\
\hline Self-worth & $43.55(33.46)$ & $63.55(31.79)$ & $30.23(31.07)$ & $31.46(32.01)$ & 0.14 & 0.10 & 0.19 & 0.08 \\
\hline Depressive symptoms & $12.82(3.25)$ & $9.82(5.38)$ & $15.85(8.32)$ & $12.23(8.42)$ & $<0.01$ & 0.31 & 0.77 & $<0.01$ \\
\hline Internalizing symptoms & $23.73(11.83)$ & $21.27(10.89)$ & $25.38(9.72)$ & $25.92(9.94)$ & 0.48 & 0.02 & 0.27 & 0.06 \\
\hline Externalizing symptoms & $12.64(7.23)$ & $9.09(3.27)$ & $16.31(6.98)$ & $16.31(8.24)$ & 0.04 & 0.18 & 0.04 & 0.18 \\
\hline
\end{tabular}

\section{Discussion}

This is the first study that explores treatment of self-esteem in youth with ASD. In a pilot RCT we explored whether COMET adds to CAU in improvement of self-esteem and self-worth in youth with ASD and whether co-occurring internalizing- and externalizing symptoms would decrease in accordance. Our results showed that the combination COMET $+\mathrm{CAU}$ caused greater improvement than CAU only in parent-reported self-esteem and co-occurring externalizing problems with large effect sizes. Self-reported self-esteem and depressive symptoms improved equally in both groups, and self-worth and parentreported internalizing symptoms did not improve. All improvements remained stable to follow-up 7 weeks after treatment, with depression improving even further.

This pilot study supports earlier findings showing that youth with ASD can benefit from psycho-social treatment (Lang, Regester, Lauderdale, Ashbaugh, \& Haring, 2010; Sukhodolsky, Bloch, Panza, \& Reichow, 2013; Weston, Hodgekins, \& Langdon, 2016) and also shows small additive effects of COMET as compared to CAU-only. However, these additional effects were mainly reported by parents (on their child's self-esteem and externalizing symptoms). Based on cut-off scores of a large Finnish sample of 15 -year olds, in this study $75 \%$ of the participants had a clinical low self-esteem according to parent-reports, while only $25 \%$ selfreported a clinical low self-esteem (Isomaa et al. 2013). Hence, there was actually little to no room for improvement from the child's perspective. Still, parents do seem to remark improvements and this can be very valuable for both parents and child. Low self-esteem and co-occurring symptoms do not only affect the child, but also parents and the family, as is seen in a lower quality of life, higher parenting stress and more depressive symptoms in these parents (Broquere et al., 2016; Lee, Harrington, Louie, \& Newschaffer, 2008; Van Steijn, Oerlemans, Van Aken, Buitelaar, \& Rommelse, 2014). So, when parents notice their child to have a more positive self-esteem and less externalizing symptoms the stress perceived in parenting might diminish and parent-child interactions may improve, which in 
turn can initiate positive effects on child functioning and even self-perceived self-esteem.

Still, the informant-discrepancy found in this study is remarkable. One might argue that differences in perspectives could be explained by the lack of ability for youth with ASD to report on self-questionnaires, since critical self-reflection is needed to do so. However, the self-reports did have high internal consistency over all three assessments, indicating that the informantdiscrepancy cannot be attributed to low reliability of self-reports (McCauley et al., 2017). Yet, it might be possible that the questionnaires used in this study are not sensitive for youth with ASD. Knowing that some children with ASD have difficulties with abstract reasoning and selfevaluations in particular, the validity of the instruments can be questioned (Henderson et al., 2009; Lombardo, Barnes, Wheelwright, \& BaronCohen, 2007; Toichi et al., 2002). Still, De Los Reyes and Kazdin (2005) mention in their work that informant discrepancies should not be only interpreted as methodological error, but rather should be used to try to understand the nature of parent-child interactions and how this in turn can affect child functioning and behavior. Therefore, we advise the use of both self- and parent-reported self-esteem and co-occurring symptoms, as both perspectives are important for assessment of treatment effects.

Another explanation, for not finding additional effects of COMET $+\mathrm{CAU}$ on self-reported selfesteem and depressive symptoms, are the characteristics of our comparison group. Although COMET has been specially designed to tackle low self-esteem and self-worth, CAU can be clientadjusted; meaning that in these particular cases CAU could already be, as much as COMET, focused on enhancing low self-esteem and selfworth and also internalizing symptoms. When CAU is already a suitable intervention, it is not surprising that no additional effects could be found. Another explanation could be the presence of a so called "waiting-list effect": participants in the CAU-only condition knew in advance that they would receive COMET $+\mathrm{CAU}$ after 7 weeks of CAU-only. A recent study (Ahola et al., 2017) showed that the expectancy of receiving psychotherapy for depression in the near future already caused depressive symptoms to decrease before therapy was induced. This could explain the improvements in self-esteem and depressive symptoms in the CAU-only group.

Our results suggest that further testing of the COMET protocol is warranted, including effectiveness studies. Participants showed high treatment fidelity and additive improvements are present in self-esteem and co-occurring externalizing symptoms. However, a limitation of this study, and at the same time another explanation for the lack of additional effects of COMET, is the lack of statistical power. Despite active recruitment of participants, we did not reach the required sample size for this study. Results should therefore be interpreted with caution. Looking at the effect sizes in this study, it could be speculated that additional effects of COMET might be present but could not be revealed due to our small sample size. Hence, it is necessary to replicate this pilot study in a larger randomized controlled trial with a power of at least .80 with a longer follow-up period, so that existing effects can be revealed. In addition, COMET in its current form is a relatively short CBT of only 7 sessions. Possibly a greater intensity of treatment (e.g. more sessions, longer sessions or more involvement of parents) is needed to enhance its effects in youth with ASD. It could take more time than 7 weeks to internalize treatment and practice newly learned skills to generalize into daily life. The improvement of depressive symptoms during the follow-up period could be an example thereof.

\section{Implications}

Taken together, this was the first randomized controlled study looking at the efficacy of COMET when added to CAU for improving low self-esteem in youth with ASD. The results of this pilot study are hopeful, as only 7 weeks of treatment caused significantly greater improvements in parent-reported self-esteem and co-occurring externalizing problems in youth with ASD. These results also support earlier found associations between low self-esteem and externalizing problems in youth with ASD (Van 
der Cruijsen \& Boyer, under revision) and suggest that treatment of self-esteem might also alleviate these externalizing problems. The results of this pilot study do not allow us to declare COMET as being necessary in enhancing low self-esteem in ASD, but we can suggest that when given parallel to CAU, COMET helps to improve self-esteem and co-occurring externalizing problems in youth with ASD in only a short period of time.

\section{References}

Achenbach, T. M. (1994). Child Behavior Checklist and related instruments. In M. E. Maruish (Ed.), The use of psychological testing for treatment planning and outcome assessment (pp. 517-549). Hillsdale: Lawrence Erlbaum Associates, Inc.

Ahola, P., Joensuu, M., Knekt, P., Lindfors, O., Saarinen, P., Tolmunen, T., Valkonen-Korhonen, M., Jääskeläinen, T., Virtala, E., Tiihonen, J., \& Lehtonen, J. (2017). Effects of scheduled waiting for psychotherapy in patients with major depression. Journal of Nervous and Mental Disease, 205(8), 611-617. doi: 10.1097/NMD.0000000000000616.

American Psychiatric Association (2000). Diagnostic and statistical manual of mental disorders (4th edition TextRevision). Washington, DC: American Psychiatric Association.

American Psychiatric Association (2013). Diagnostic and statistical manual of mental disorders (5th edition). Washington, DC: American Psychiatric Association.

Ankenman, K., Elgin, J., Sullivan, K., Vincent, L., \& Bernier, R. (2014). Nonverbal and verbal cognitive discrepancy profiles in Autism Spectrum Disorders: Influence of age and gender. American Journal on Intellectual and Development and Disabilities, 119(1), 84-99. https://doi.org/10.1352/1944-7558-119.1.84

Blascovich, J., \& Tomaka, J. (1991). Measures of selfesteem. In J. P. Robinson, P. R. Shaver, \& L. S. Wrightsman (Eds.), Measures of social psychological attitudes, Vol. 1. Measures of personality and social psychological attitudes (pp. 115-160). San Diego, CA, US: Academic Press.

Brewin, C.R. (2006). Understanding cognitive behaviour therapy: A retrieval competition account. Behaviour Research and Therapy, 44(6), 765-784. doi: 10.1016/j.brat.2006.02.005

Broquere, M., Soussana, M., Michelon, C., Rattaz, C., Brisot, J., \& Baghdadli, A. (2016). Impact of anxiety disorders on quality of life of adolescents with autism spectrum disorder without intellectual disability.

L'Encephale, 42(6), 499-505. doi: 10.1016/j.encep.2015.12.025

Ekkers, W., Korrelboom, K., Huijbrechts, I., Smits, N., Cuijpers, P., \& van der Gaag, M. (2011). Competitive Memory Training for treating depression and rumination in depressed older adults: A randomized controlled trial.
Behaviour Research and Therapy, 49(10), 588-596. doi: 10.1016/j.brat.2011.05.010.

Estes, A., Rivera, V., Bryan, M., Cali, P., \& Dawson, G. (2011). Discrepancies between academic achievement and intellectual ability in higher-functioning school-aged children with autism spectrum disorder. Journal of Autism and Developmental Disorders, 41(8), 1044-1052. http://doi.org/10.1007/s10803-010-1127-3.

Everaert, J., Koster, E. H. W., Schacht, R., \& De Raedt, R. (2010). Evaluatie van de psychometrische eigenschappen van de Rosenberg zelfwaardeschaal in een poliklinische psychiatrische populatie. Gedragstherapie, 43(4), 307317.

Fournier, K. A., Hass, C. J., Naik, S. K., Lodha, N., \& Cauraugh, J. H. (2010). Motor coordination in Autism Spectrum Disorders: A synthesis and meta-analysis. Journal of Autism and Developmental Disorders, 40, 1227-1240. DOI: 10.1007/s10803-010-0981-3

Frith, U. (2003). Autism: Explaining the enigma (2nd ed.). Malden: Blackwell Publishing

De Los Reyes, A., \& Kazdin, A. E. (2005). Informant discrepancies in assessment of childhood psychopathology: A critical review, theoretical framework, and recommendations for further study. Psychological Bulletin, 131(4), 482-509. doi:10.1037/0033-2909.131.4.483

Harter, S. (1985). Manual for the self-perception profile for children. Denver, CO: University of Denver.

Harter, S. (1988). Manual for the self-perception profile for adolescents. Denver, CO: University of Denver

Harter, S. (2012). The construction of the self. New York, NY: Guilford Press.

Harter, S., Waters, P., \& Whitesell, N. R. (1998). Relational self-worth: Differences in perceived worth as a person across interpersonal contexts among Adolescents. Child Development, 69(3), 756-766. doi: 10.1111/j.14678624.1998.tb06241.x

Hawker, D. S., \& Boulton, M. J. (2000). Twenty years ' research on peer victimization and psychosocial maladjustment : A meta-analytic review of crosssectional studies. The Journal of Child Psychology and Psychiatry, 41(4), 441-455. doi: 10.1111/14697610.00629

Henderson, H. A., Zahka, N. E., Kojkowski, N. M., Inge, A. P., Schwartz, C. B., Hilleman, C. M., Coman, D. C., \& Mundy, P. C. (2009). Self-referenced memory, social cognition, and symptom presentation in autism. Journal of Child Psychology and Psychiatry, 50(7), 853-861. doi: 10.1111/j.1469-7610.2008.02059.x.

Holmes, E. A., Mathews, A., Mackintosh, B., \& Dalgleish, T. (2006). The causal effect of mental imagery on emotion assessed using picture-word cues. Emotion, 8(3), 395409. doi: 10.1037/1528-3542.8.3.395.

Isomaa, R., Väänänen, J., Fjörd, S., Kaltiala-Heino, \& R., Marttunen, M. (2013). How low is low? Low self-esteem as an indicator of internalizing psychopathology in adolescence. Health Education \& Behavior, 40(4), 392399. doi: https://doi.org/10.1177/1090198112445481 
Joseph, R. M., Tager-Flusberg, H., \& Lord, C. (2002). Cognitive profiles and social-communicative functioning in children with autism spectrum disorder. Journal of Child Psychology and Psychiatry, 43(6), 807-821. doi: 10.1111/1469-7610.00092.

Kort, W., Schittekatte, M., Bosmans, M., Compaan, E., Vermeer, G., \& Verhaeghe, P. (2005). Wechsler Intelligence Scale for Children-III: Handleiding. Amsterdam: Pearson.

Korrelboom, K., De Jong M., Huijbrechts, I., \& Daansen, P. (2009). Competitive Memory Training (COMET) for treating low self-esteem in patients with eating disorders: A randomized clinical trial. Journal of Consulting and Clinical Psychology, 77(5), 974-980. doi: 10.1037/a0016742.

Korrelboom, K., Maarsingh, M., \& Huijbrechts, I. (2012). Competitive Memory Training (COMET) for treating low self-esteem in patients with depressive disorders: A randomized clinical trial. Depression and Anxiety, 29(2), 102-110. doi: 10.1002/da.20921.

Korrelboom, K., Van der Weele, K., Gjaltema, M., \& Hoogstraten, C. (2009). Competitive Memory Training for treating low self-esteem: A pilot study in a routine clinical setting. The Behavior Therapist, 32(1), 3-8. doi: https://doi.org/10.1017/S1352465810000469

Kuin, M., \& Peters, P. (2014). Zelfbeeldtraining voor kinderen en jongeren: Competitive Memory Training (COMET). Houten Bohn Stafleu van Loghum.

Kuin, M., Peters, P., Galesloot, E., Van Steensel, B., \& Bögels, S. (in preparation.) The effectiveness of a selfesteem training for children and adolescents. Manuscript submitted for publication.

Kwok, E. Y. L., Brown, H. M., Smyth, R. E., \& Cardy, J. O. (2015). Meta-analysis of receptive and expressive language skills in autism spectrum disorder. Research in Autism Spectrum Disorders, 9, 202-222. https://doi.org/10.1016/j.rasd.2014.10.008

Lang, P. J. (1985). The cognitive psychophysiology of emotion: Fear and anxiety. In A. H. Tuma, \& J. Maser (Eds.), Anxiety and the anxiety disorders (pp. 131-171). Hillsdale, New Jersey, London: Lawrence Erlbaum Associates, Publishers.

Lang, R., Regester, A., Lauderdale, S., Ashbaugh, K., \& Haring, A. (2010). Treatment of anxiety in autism spectrum disorders using cognitive behaviour therapy: A systematic review. Developmental Neurorehabilitation, 13(1), 53-63. doi: 10.3109/17518420903236288

Lee, L. C., Harrington, R. A., Louie, B. B., \& Newschaffer, C. J. (2008). Children with autism: Quality of life and parental concerns. Journal of autism and developmental disorders, 38(6), 1147-1160. doi: 10.1007/s10803-0070491-0

Lombardo, M. V., Barnes, J. L., Wheelwright, S. J., \& Baron-Cohen, S. (2007). Self-referential cognition and empathy in autism. PLoS One, 2(9), e883. doi: 10.1371/journal.pone.0000883.

Macmullen-Freeman, L., Locke, J., Rotheram-Fuller, E., \& Mandell, D. (2017). Brief report: Examining executive and social functioning in elementary-aged children with autism. Journal of Autism and Developmental Disorders, 47(6), 1890-1895. doi: 10.1007/s10803-017-3079-3.

Mann, M., Hosman, C. M. H., Schaalma, H. P., \& de Vries, N. K. (2004). Self-esteem in a broad-spectrum approach for mental health promotion. Health Education Research, 19(4), 357-372. doi: 10.1093/her/cyg041

Matson, J. L., \& Nebel-Schwalm, M. S. (2007). Comorbid psychopathology with autism spectrum disorder in children: An overview. Research in Developmental Disabilities, 28(4), 341-352.

doi: 10.1016/j.ridd.2005.12.004

McCauley, J. B., Harris, M. A., Zajic, M. C., Swain-Lerro, L. E., Oswald, T., McIntyre, N., Trzesniewski, K., Mundy, P., \& Solomon, M. (2017). Self-esteem, internalizing symptoms, and Theory of Mind in youth with autism spectrum disorder. Journal of Clinical Child and Adolescent Psychology, 00(00), 1-12. doi: 10.1080/15374416.2017.1381912.

Ming, X., Brimacombe, M., \& Wagner, G. C. (2007). Prevalence of motor impairment in autism spectrum disorders. Brain and Development, 29(9), 565-570. https://doi.org/10.1016/j.braindev.2007.03.002

Ozonoff, S. \& Jensen, J. (1999). Specific executive function profiles in three neurodevelopmental disorders. Journal of Autism and Developmental Disorders, 29(2), 171-177. http://dx.doi.org/10.1023/A:1023052913110

Ozonoff, S., Strayer, D. L., McMahon, W. M., \& Filloux, F. (1994). Executive function abilities in autism and tourette syndrome: An information processing approach. The Journal of Child Psychology and Psychiatry, 35(6), 1015-1032.

http://dx.doi.org/10.1111/j.1469-7610.1994.tb01807.x

Portway, S., \& Johnson, B. (2003). Asperger syndrome and the children who "Don"t quite fit in'. Early Child Development and Care, 173(4), 435-443. https://doi.org/10.1080/0300443032000079113

Posthuma, D., \& Lange, A. (1999). Positieve zelfverbalisatie bij opgenomen patiënten: Een pilot-onderzoek. Directieve Therapie, 19(3), 51-57. doi:10.1007/BF03060211

Reijntjes, A., Thomaes, S, Kamphuis, J. H., Orobio de Castro, B., \& Telch, M. J. (2010). Self-verification strivings in children holding negative self-views: The mitigating effects of a preceding success experience. Cognitive Therapy and Research, 34(6), 563-570. doi: 10.1007/s10608-009-9289-Z

Roeyers, H., Thys, M., Druart, C., De Schrijver, M., \& Schittekatte, M. (2011). SRS -2 Screeningslijst voor autismespectrumstoornissen. Amsterdam: Hogrefe Uitgevers BV.

Rosenberg, M. (1979). Components of Rosenberg's selfesteem scale. Conceiving the self. New York: Basic Books.

Sattler, J. M. (2001). Assessment of Children: Cognitive Applications (4th edition). San Diego, CA.

Schmitt, D. P., \& Allik, J. (2005). Simultaneous administration of the Rosenberg Self-Esteem Scale in 53 Nations: Exploring the universal and culture-specific features of global self-esteem. Journal of Personality and 
Social Psychology, 89(4), 623-642. doi:10.1037/00223514.89.4.623

Schneider, B. C., Wittekind, C. E., Talhof, A., Korrelboom, K., \& Moritz, S. (2015). Competitive Memory Training (COMET) for OCD: A self-treatment approach to obsessions. Cognitive Behaviour Therapy, 44(2), 142152. doi: 10.1080/16506073.2014.981758

Schuh, J. M., \& Eigsti, I. (2012). Working memory, language skills, and autism symptomatology. Behavorial Sciences, 2(4), 207-218. doi: 10.3390/bs2040207

Simonoff, E., Pickels, A., Charman, T., Chandler, S., Loucas, T., \& Baird, G. (2008). Psychiatric disorders in children with autism spectrum disorders: Prevalence, comorbidity, and associated factors in a population-derived sample. Journal of the American Academy of Child and Adolescent Psychiatry, 47(8), 921-929. doi: 10.1097/CHI.0b013e318179964f.

Sitarenios, G., \& Kovacs, M. (1999). Use of the Children's Depression Inventory. In M. E. Maruish (Ed.), The use of psychological testing for treatment planning and outcomes assessment (pp. 267-298). Mahwah, NJ, US: Lawrence Erlbaum Associates Publishers.

Sowislo, J. F., \& Orth, U. (2013). Does low self-esteem predict depression and anxiety? A meta-analysis of longitudinal studies. Psychological Bulletin, 139(1), 213240. doi: 10.1037/a0028931

Staring, A. B. P., van den Berg, D. P. G., Cath, D. C., Schoorl, M., Engelhard, I. M., \& Korrelboom, K. (2016). Self-esteem treatment in anxiety: A randomized controlled crossover trial of Eye Movement Desensitization and Reprocessing (EMDR) versus Competitive Memory Training (COMET) in patients with anxiety disorders. Behaviour Research and Therapy, 82, 11-20. doi: 10.1016/j.brat.2016.04.002.

Sukhodolsky, D. G., Bloch, M. H., Panza, K. E., \& Reichow, B. (2013). Cognitive-behavioral therapy for anxiety in children with high-functioning autism: A meta-analysis. Pediatrics, 132(5), 1341-1350. doi: 10.1542/peds.20131193.

Timbremont, B., \& Braet, C. (2008). Children's Depression Inventory Handleiding. Amsterdam: Pearson.

Toichi, M., Kamio, Y., Okada, T., Sakihama, M., Youngstrom, E. A., Findling, R. L., \& Yamamoto, K. (2002). A lack of self-consciousness in autism. American Journal of Psychiatry, 159(8), 1422-1424. doi: 10.1176/appi.ajp.159.8.1422

Treffers, A., Goedhart, A. W., Veerman, J. W., Van den Bergh, B. R. H., Ackaert, L., \& De Rycke, L. (2002). Competentie Belevings-Schaal voor Adolescenten. Handleiding. Lisse: Swets Test Publishers.

Tuijl, L. A., de Jong, P. J., Sportel, B. E., de Hulle, E., \& Nauta, M. H. (2014). Implicit and explicit self-esteem and their reciprocal relationship with symptoms of depression and social anxiety: A longitudinal study in adolescents. Journal of Behavior Therapy and Experimental Psychiatry, 45(1), 113-121. https://doi.org/10.1016/j.jbtep.2013.09.007
Van der Cruijsen, R. \& Boyer, B. E. (under review). Implicit and explicit self-esteem in youth with Autism Spectrum Disorders. Manuscript submitted for publication.

Van der Does, W. (2002). Different types of experimentally induced sad mood? Behavior Therapy, 33(4), 551-561. https://doi.org/10.1016/S0005-7894(02)80016-8

Van der Gaag, M., Van Oosterhout, B., Daalman, K., Sommer, I. E., \& Korrelboom, K. (2012). Initial evaluation of the effects competitive memory training (COMET) on depression in schizophrenia-spectrum patients with persistent auditory verbal hallucinations: A randomized controlled trial. The British Journal of Clinical Psychology, 52(2), 158-171. doi: 10.1111/j.2044-8260.2011.02025.x.

Van Steijn, D. J., Oerlemans, A. M., Van Aken, M. A., Buitelaar, J. K., \& Rommelse, N. N. (2014). The reciprocal relationship of $\mathrm{ASD}$, ADHD, depressive symptoms and stress in parents of children with ASD and/or ADHD. Journal of autism and developmental disorders,44(5), 1064-1076. doi: 10.1007/s10803-013-1958-9

Veerman, J. W., Straathof, M. A. E., Treffers, A., Van den Bergh, B. R. H., \& Ten Brink, L. T. (1997). Competentiebelevingsschaal voor Kinderen (CBSK): Handleiding. Lisse: Swets \& Zeitlinger

Verhulst, F. C., Van der Ende, J., \& Koot, H. M. (1996). Handleiding voor de CBCL 4-18 (Nederlandse versie). Rotterdam: Afdeling Kinder- en Jeugdpsychiatrie, Sophia Kinderziekenhuis/Academisch Ziekenhuis/Erasmus Universiteit.

Westenberg, P. M., Drewes, M. J., Goedhart, A. W., Siebelink, B. M., \& Treffers, P. D. (2004). A developmental analysis of self-reported fears in late childhood through mid-adolescence: Social-evaluative fears on the rise? Journal of Child Psychology and Psychiatry, 45(3), 481-495. https://doi.org/10.1111/j.1469-7610.2004.00239.x

Weston, L., Hodgekins, J., \& Langdon, P. E. (2016). Effectiveness of cognitive behavioral therapy with people who have autistic spectrum disorders: A systematic review and meta-analysis. Clinical Psychology Review, 49, 41-54. doi: 10.1016/j.cpr.2016.08.001.

White, S. W., Oswald, D., Ollendick, T., \& Scahill, L. (2009). Anxiety in children and adolescents with autism spectrum disorders. Clinical Psychology Review, 29(3), 216-229. doi: 10.1016/j.cpr.2009.01.003.

World Health Organization (2017). Autism spectrum disorders, 4 April 2017. https://www.who.int/newsroom/fact-sheets/detail/autism-spectrum-disorders. 